
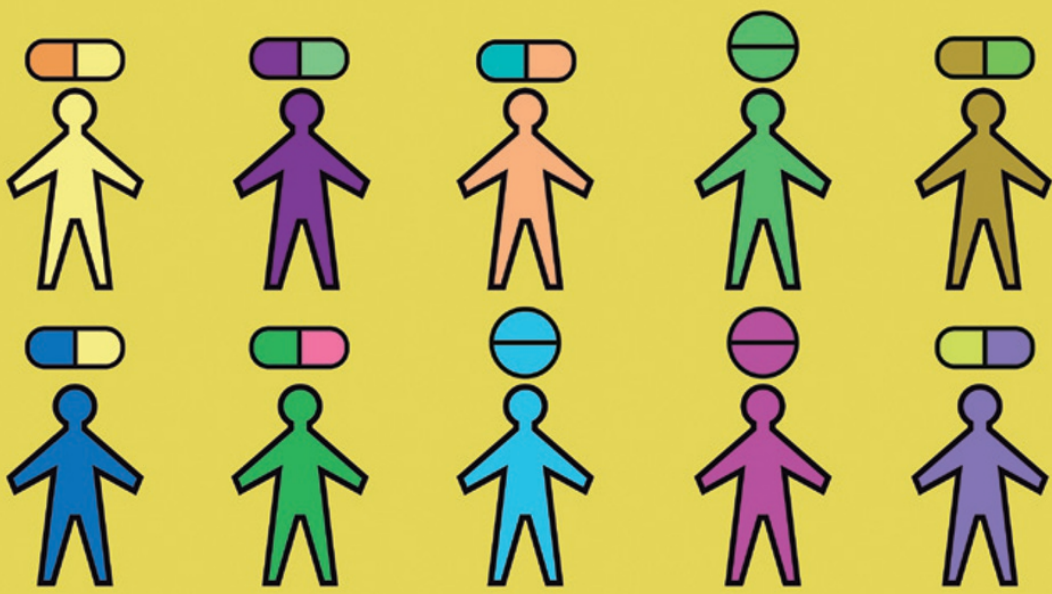

TRIAL WATCH

\section{Lung cancer trial aims to bolster personalized medicine}

The charity Cancer Research UK (CRUK) has teamed up with AstraZeneca and Pfizer to launch a clinical trial for lung cancer that tests several targeted anticancer therapies in a single trial. The trial — dubbed the National Lung Matrix Trial — aims to increase the efficiency of developing personalized therapies and delivering these to patients.

In the trial, which is anticipated to begin recruiting late this summer, groups of 20-30 patients with stage 3 or more advanced non-small-cell lung cancer will be matched to a specific drug (or, potentially, drug combinations) based on the genotype of their tumour. Initially, there will be 14 different stratifications of 6 drugs (TABLE 1).

John Gosney, professor of thoracic pathology at the University of Liverpool, UK (and an advisor to CRUK), highlights that the number of genetic abnormalities that can be currently targeted is low. "There are significant problems of scale, logistics and cost when profiling tumour tissues," he says. "The Matrix trial's centralized and comprehensive genetic analysis of tumours from a large number of patients is a huge step forward - robust data will be able to be generated quickly and efficiently."

If a particular drug treatment shows promise in the trial - which will be defined by improvements in patient survival, tumour shrinkage or an alleviation of symptoms then the arm could potentially break away from the main trial and move into a larger independent trial. And arms that do not show early signs of benefit can be stopped.

This design moves away from the traditional context of 'one drug, one trial'. lan Walker, Head of Stratified Medicine at CRUK, notes that this old approach is expensive and wasteful from a pharmaceutical perspective and challenging from a patient perspective (as patients have to undergo testing to find out whether their genotype is suitable for each specific trial). "It's clearly not in anyone's interest to go on developing drugs in this inefficient way," he says.

Donald Berry, a professor in the Department of Biostatistics at the University of Texas MD Anderson Cancer Center, Houston, USA, praises CRUK's efforts and agrees that the Matrix trial will improve the logistics of clinical drug testing, but feels that some aspects of the trial's design could be more innovative. "CRUK and its partners assume that the drug's targets are known, yet research into cancer and drug development has taught us that agents may hit targets that are in addition to or instead of their putative targets." He adds that one way to address the possibility of off-target effects - which could be detrimental or beneficial - is to use a trial design that incorporates adaptive randomization in order to focus on the responding patient population (whereby the initial randomization of drugs considers all genotypes, then as the trial generates results, patients are randomized to drugs that the accumulating evidence indicates will benefit them).

Berry also notes that early indicators of a drug's effect, such as tumour shrinkage, don't always translate into clinical benefit, and indeed it may be difficult to measure end points of improved survival or symptoms in small groups of patients.

But Walker notes that Matrix is a "signal-finding trial" to see whether the drugs show promise in stratified patient groups, and will be used to decide whether or not to move drugs into larger randomized trials. "The statistically powered analysis will be done in Phase II trials," he says, adding that "potential off-target effects will not lessen or invalidate this very innovative approach to delivering targeted medicines".

As well as the six initial drugs, there will be the potential to add further drugs during the course of the trial, as such drugs become available from AstraZeneca's and Pfizer's pipelines. Pharmaceutical companies are cooperating more and more, and improved trial design could lead to substantial economies of scale; indeed, CRUK is also talking to other companies about their development portfolios. "It will be a really exciting development to take other drugs on board as we go forward," enthuses Walker.

Charlotte Harrison

\section{Table 1 | Drugs that will be included in the initial stage of the Matrix trial}

\begin{tabular}{lll} 
Drug & Mechanism of action & Highest phase \\
\hline Crizotinib & ALK inhibitor & Launched \\
\hline Palbociclib & CDK4 and CDK6 inhibitor & Phase III \\
\hline Selumetinib & MEK1 and MEK2 inhibitor & Phase III \\
\hline AZD4547 & FGFR antagonist & Phase II \\
\hline AZD2014 & mTOR inhibitor & Phase II \\
\hline MEDI4736 & PDL1 inhibitor & Phase II \\
\hline
\end{tabular}

ALK, anaplastic lymphoma kinase; CDK, cyclin-dependent kinase; FGFR, fibroblast growth factor receptor; MEK; MAPK/ERK kinase; mTOR, mammalian target of rapamycin; PDL1, programmed cell death 1 ligand 1. 